José Goyanes. Aportaciones experimentales a la cirugía vascular

\title{
José Goyanes. Experimental contributions to vascular surgery
}


José Goyanes. Aportaciones experimentales a la cirugía vascular

José Goyanes. Experimental contributions to vascular surgery

Francisco S. Lozano Sánchez ${ }^{1}$, Luis M. Reparaz Asensio²

${ }^{1}$ Servicio de Angiología y Cirugía Vascular. Hospital Universitario de

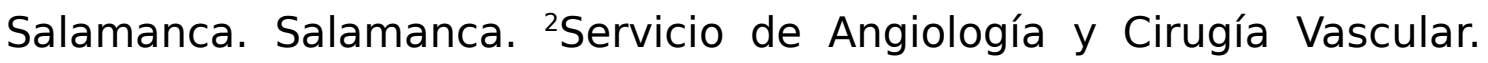
Hospital General Universitario Gregorio Marañón. Madrid

Correspondencia: Francisco S. Lozano Sánchez. Servicio de Angiología y Cirugía Vascular. Complejo Asistencial Universitario de Salamanca. Paseo de San Vicente, s/n.

37007 Salamanca.

e-mail: lozano@usal.es

Palabras clave: Injertos vasculares. Cirugía experimental. Cirugía vascular.

Keywords: Vascular graft. Experimental surgery. Vascular surgery.

Recibido: $16 / 05 / 2020$

Aceptado: 22/05/2020

Recientemente hemos tenido la oportunidad de leer los originales de algunos trabajos de investigación experimental escritos por el profesor Goyanes. Esas publicaciones, que ya conocíamos a través de las referencias de otros autores, cambian su significado al leer el original (con su redacción y estilo) y aportan una visión enriquecedora que deseamos compartir con los lectores de esta nota histórica.

En 1905, Goyanes publicó en la Revista de Medicina y Cirugía Prácticas un estudio experimental sobre la cirugía de los vasos (Fig. 1). En diversos capítulos trató temas como las causas de la formación de trombos en los vasos suturados, la reparación o la cicatrización de 
las heridas arteriales y venosas. Un capítulo trató específicamente la vitalidad de las paredes vasculares denudadas y de la trasplantación o injerto vascular (1).

En aquellos tiempos se creía que un vaso denudado y aislado (posteriormente denominado injerto vascular) se necrosaría al no estar vascularizado. En este contexto, Goyanes cita los experimentos de Höpfner (2), quien trasplantó con éxito en un perro "un trozo de carótida en la arteria femoral, después de resecar una porción de este vaso. A las ocho semanas, el vaso trasplantado estaba permeable, su pared algo engrosada y la túnica interna intacta". El mismo resultado lo obtuvo trasplantando la femoral en la carótida.

Asimismo, consiguió trasplantar con éxito la femoral de un perro en la carótida de otro; por el contrario, los trasplantes arteriales entre animales de distintas especies no obtuvieron buenos resultados. En cuanto a los trasplantes de venas, los resultados tampoco fueron exitosos (trombosis). El mismo Goyanes insertó en un perro un segmento de la cava en la aorta que finalizó con hemorragia al cuarto día. Estos últimos resultados son contradictorios con los obtenidos en el ser humano, como posteriormente comentaremos.

Concluye Goyanes diciendo textualmente: "No necesito encomiar el porvenir clínico de las transplantaciones vasculares. En el hombre podría ensayarse la sustitución plástica de un segmento arterial, perdido por lesión traumática o morbosa, por otro segmento, tomado de un cadáver reciente".

Como curiosidad adicional, para darnos cuenta de en qué época nos encontramos, hay que referir que la Revista de Medicina y Cirugía Prácticas, fundada en 1877 y que tenía una periodicidad semanal, costaba 1 peseta por número (la subscripción anual ascendía a 20 pesetas).

Con estos y otros precedentes, Goyanes interpuso la vena poplítea ipsilateral como reemplazo del defecto ocasionado por la resección de un gran aneurisma poplíteo (fusiforme y de origen arteriosclerótico o sifilítico). La operación se realizó el 12 de junio de 1906 en el Hospital 
General de Madrid. El caso clínico (táctica y técnica) fue publicado el 8 de septiembre del mismo año en la revista El Siglo Médico (3). Como esta operación ha sido comentada y resaltada por numerosos autores españoles y extranjeros, solo cabe recordar que la historia la contempla como el primer injerto venoso empleado en cirugía humana. La bibliografía médica la denomina como técnica de Goyanes o Goyanes-Lexer.

\section{JÓSE GOYANES CAPDEVILLA (1876-1964)}

Nació en Monforte de Lemos (Lugo) el 16 de junio de 1876. Estudió Medicina en Madrid (se licenció con honores en 1900 y se doctoró con premio extraordinario en 1901). Cuatro años después (1905) obtuvo por oposición el puesto de jefe de un servicio de cirugía del Hospital General de Madrid (hoy, Hospital General Universitario Gregorio Marañón). Posteriormente, y siendo profesor auxiliar de Patología Quirúrgica en el Hospital de San Carlos de Madrid, opositó a la cátedra que había dejado vacante su maestro (Alejando San Martín, otro gigante de la cirugía vascular española, quien inculcó a su discípulo, entre otras, la preferencia por la cirugía vascular y la experimentación), pero, injustificadamente y por oscuros motivos, no obtuvo la plaza. Fue director del entonces Instituto Príncipe de Asturias (posteriormente Instituto del Cáncer de Madrid) hasta 1931, cuando fue sustituido por motivos políticos.

Durante la Guerra Civil estuvo en Salamanca practicando cirugía de guerra. Posteriormente regresó a Madrid (hasta 1945) y, finalmente, se marchó a Santa Cruz de Tenerife por problemas familiares, donde falleció en 1964 (88 años). En Canarias ocupó su tiempo publicando artículos no quirúrgicos (históricos y de otro tipo).

El Dr. Goyanes practicó más de 12000 intervenciones de todo tipo y fundó un departamento de cirugía experimental que costeó él mismo. Publicó innumerables trabajos e ideó técnicas e instrumental de gran precisión, utilidad y eficacia. 
Fue presidente de la Academia Médico-Quirúrgica Española, presidente de la Asociación Española de Cirujanos, socio de honor de la Academia Médica de Guipúzcoa, de la Sociedad Internacional de Cirugía y de la Sociedad de Cirujanos de Bruselas. Doctor honoris causa por la Facultad de Medicina de Burdeos y miembro del Colegio de Cirujanos de Norteamérica. Poseía la Gran Cruz de Alfonso XII, la Legión de Honor y fue caballero de la Orden de Santiago y de la Espada de Portugal. Ocupó el sillón 41 de la Real Academia de Medicina.

Para concluir, hay que resaltar que Goyanes, además de un gran cirujano clínico e investigador, fue un humanista. Como botón de muestra, basta referir que conocía numerosos idiomas (incluidos el latín y griego) y que fue amigo de las grandes figuras vasculares (Matas, los hermanos Mayo, Carrel, etc.) y de intelectuales (Unamuno, Ortega, Marañón, etc.) de su época (Fig. 2).

\section{BIBLIOGRAFÍA}

1. Goyanes J. Sobre angioplastia. Contribución al estudio experimental de la cirugía de los vasos. Revista de Medicina y Cirugía Prácticas 1905;879:89-96.

2. Höpfner E. Ueber Gefässnaht, Gefässtransplantationen und Replantation von amputirten Extremitäten. Archiv für Klinische Chirurgie 1903;LXI-LXX:417-71.

3. Goyanes J. Sustitución plástica de las arterias por las venas o arterioplastia venosa aplicada como nuevo método al tratamiento de los aneurismas. El Siglo Médico 1906;2752:561-4.

\section{PIES DE FIGURAS}

Figura 1. Primera página del artículo de José Goyanes “Sobre angioplastia. Contribución al estudio experimental de la cirugía de los vasos", en la Revista de Medicina y Cirugía Prácticas (año 1905).

Figura 2. José Goyanes Capdevilla (1876-1964) junto a Miguel de Unamuno y Jugo (1864-1936). 